\title{
DETERMINANTES DEL ÉXITO EN LA FORMULACIÓN DE DECISIONES ESTRATÉGICAS EN INSTITUCIONES UNIVERSITARIAS
}

\section{Introducción}

Las decisiones estratégicas constituyen elecciones cuyo impacto es de largo alcance; por lo tanto, son decisiones no rutinarias que involucran recursos significativos de las organizaciones, implican la participación de varios niveles y funciones al interior de las instituciones y pueden conducir al éxito o fracaso.

La toma de decisiones estratégicas es un proceso vital de la dirección estratégica, ya que es la instancia en la cual las organizaciones seleccionan sus mercados, eligen su posición competitiva y construyen sus competencias esenciales.

No obstante su importancia, se ha comprobado que casi el 50\% de las decisiones fracasan; en consecuencia, su diseño e implementación es una tarea fundamental para los equipos de alta dirección.

Las universidades constituyen un tipo de organización con una estructura organizativa similar a las burocracias profesionales. El entorno de las universidades es complejo y se ha ido tornando más dinámico y hostil en los últimos años. Las instituciones no tienen un criterio orientador único, sino múltiples objetivos que pueden entrar en conflicto. En ellas existen múltiples influencias internas y externas, así como diversas fuentes de financiamiento.

En este marco, ciertas universidades son más exitosas que otras. Aquellas cuyas decisiones estratégicas logran mejor calidad alcanzan mayores niveles de eficacia. Conocer y comprender las variables que influyen en el diseño de las decisiones estratégicas es una tarea fundamental cuyo resultado puede conducir a descubrir prácticas que generen mejores posibilidades de éxito estratégico en universidades. 
El objetivo de esta investigación es describir las relaciones entre el diseño de las decisiones estratégicas y el grado de racionalidad del proceso de toma de decisiones, intentando descubrir en qué medida la flexibilidad y el conflicto cognitivos pueden afectar el grado de racionalidad del proceso de toma de decisiones estratégicas. La flexibilidad cognitiva se puede entender como el grado en el cual se exploran nuevas ideas o supuestos en el proceso de toma de decisiones; el conflicto cognitivo como el grado en el cual existen posiciones o ideas diferentes entre sí en el proceso de toma de decisiones (Rodríguez Ponce, 2004).

El estudio avanza significativamente en el acervo de conocimientos sobre el tema, en tanto permite: 1) descubrir los principales determinantes del éxito en el diseño de las decisiones estratégicas; 2) proporcionar evidencia empírica en un campo disciplinario de exigencia creciente para las instituciones universitarias, y 3) generar buenas prácticas para mejorar las posibilidades de éxito en la dirección de las instituciones universitarias.

\section{Marco teórico e hipótesis}

La teoría de los escalafones superiores plantea que los resultados de la organización, es decir, las elecciones estratégicas y el desempeño, están predeterminados en forma parcial por las características del equipo de alta dirección. Este estudio se basa en la premisa de que, en un contexto de racionalidad limitada, la base cognitiva y los valores limitan el campo de observación, influyendo sobre la percepción selectiva, la interpretación y, por ende, en las percepciones de la administración y en la selección de la estrategia. Desde esta perspectiva, la creación de valor estratégico en las organizaciones se genera en los procesos de toma de decisiones estratégicas (Hambrick y Mason, 1984; Waldman et al., 2001; Carpenter et al., 2001; Hambrick, 2007).

Las decisiones estratégicas constituyen elecciones con impactos significativos de largo plazo y, por lo tanto, son decisiones que involucran el empleo de recursos importantes de las organizaciones y consideran la participación de varios niveles y funciones. De estas 
decisiones depende su éxito o fracaso (Pedraja Rejas y Rodríguez Ponce, 2008a, 2008b, Pedraja Rejas et al., 2008).

Desde luego, pueden existir decisiones que generan mejores resultados que otras. Su calidad se puede medir desde diferentes perspectivas. Sin embargo, un alto número de investigaciones enfatiza en la rigurosidad de la decisión, el número de alternativas y la calidad de las decisiones generadas, el logro de los objetivos y la creación de valor estratégico como elementos básicos para evaluar la decisión alcanzada (Hollenbeck et al., 1998; Dean y Sharfman, 1996; Valacich y Schwenk, 1995, Molloy y Schwenk, 1995, Amason, 1996, Rodríguez Ponce, 2007a; 2007b; Pedraja Rejas et al., 2008 a, 2008b). Este será el enfoque de análisis en la investigación.

Se puede esperar que mejores decisiones conduzcan a mayor eficacia de las organizaciones. Ciertamente, estudios empíricos prueban que existe una relación significativa entre calidad de las decisiones y eficacia de las organizaciones en decisiones de tipo funcional referidas, por ejemplo, a sistemas de información (Changchien y Lin, 2005; Grover y Segars, 2005), sistemas productivos (Dowlatshahi, 2005; Tapinos et al., 2005) y elecciones de marketing (Katsikeas et al., 2006, Athaide y Desai, 2005). Pero también dicha relación se ha probado en decisiones estratégicas corporativas (Hiller y Hambrick, 2005; Arendt et al., 2005). Por lo tanto, en las instituciones universitarias es factible encontrar una relación directa y positiva entre la calidad del diseño de las decisiones estratégicas y su eficacia corporativa (Rodríguez Ponce, 2006; Rodríguez Ponce y Pedraja Rejas, 2007).

No obstante, cerca del 50\% de las decisiones fracasan (Nutt, 1999). El gran desafío consiste en identificar el determinante estructural de la calidad de las decisiones. Aunque en la década de los 80 no existía claridad respecto del impacto de la racionalidad sobre la calidad de las decisiones y, por ende, sobre la eficacia de las organizaciones, en el último tiempo se ha establecido que la racionalidad se relaciona de manera positiva con la calidad de la formulación de las decisiones estratégicas en las organizaciones en general y, por cierto, puede impactar sobre el grado de éxito de las firmas (Goll y Rasheed, 1997; 2005; Miller et al., 1998; Papadakis et 
al., 1998). En el caso de las instituciones universitarias, se ha probado que mayores grados de racionalidad pueden conducir a que el equipo de alta dirección genere decisiones de mejor calidad (RodríguezPonce, 2006; Rodríguez Ponce y Pedraja Rejas, 2007).

Sin embargo, las principales investigaciones sobre la relación entre eficacia organizativa y racionalidad ignoran u omiten los determinantes de la racionalidad. Pareciera ser que el estado del arte supone una perspectiva autogenerada o endógena del grado de racionalidad de la toma de decisiones. Bajo este enfoque, la racionalidad sería un resultado del propio proceso de interacción entre los equipos de alta dirección.

Siguiendo a Sharfman y Dean (1997) y a Dean y Sharfman (1996), se podría postular que en cuanto en el proceso de toma de decisiones estratégicas 1) se confíe en nuevas fuentes de información, 2) se reciban y analicen nuevas ideas, 3) se logre una participación amplia y creativa de los altos directivos, y 4) se reconsideren las posiciones originales sería posible lograr un mayor nivel de racionalidad en la generación y selección de opciones estratégicas.

Más aún, de acuerdo con Amason (1996) y Jehn (1997), es posible plantear que, en tanto en el proceso de toma de decisiones estratégicas 1) se discutan los puntos de vista desde diferentes enfoques o perspectivas, 2) se favorezca la opción de ideas u opciones alternativas, y 3) se favorezca el intercambio de ideas u opiniones entre los participantes es posible un mayor nivel de racionalidad.

En consecuencia, las hipótesis de la investigación son las siguientes:

H-1: La racionalidad impacta positivamente sobre el diseño de las decisiones estratégicas.

Esta relación cuenta con sólida evidencia en los últimos años (Goll y Rasheed, 2005; Rodríguez Ponce, 2006; Rodríguez Ponce y Pedraja Rejas, 2007). La tesis que sustenta la relación entre racionalidad y calidad de las decisiones se asocia con que una mayor exhaustividad en la búsqueda y análisis de información permitirá 
generar y elegir mejores alternativas, lo cual también es cierto en el campo de la educación (Hatcher, 1998; De Boer et al., 2007; Leader, 2004).

H-2: La flexibilidad cognitiva impacta positivamente sobre el grado de racionalidad del proceso de toma de decisiones estratégicas.

La relación entre flexibilidad cognitiva y racionalidad ha sido prácticamente inexplorada en la literatura internacional. Sin embargo, en los últimos años se ha propuesto -aunque no necesariamente comprobado- que una mayor apertura cognitiva puede conducir a una mayor exhaustividad en los procesos decisionales (Pansiri, 2005; Rodríguez-Ponce, 2006).

H-3: El conflicto cognitivo impacta positivamente sobre el grado de racionalidad del proceso de toma de decisiones estratégicas.

La relación entre conflicto cognitivo y racionalidad no es común en los estudios internacionales. Si bien una serie de investigaciones sugiere ambas variables como componentes del proceso de toma de decisiones estratégicas (Papadakis et al, 1998; Rodríguez Ponce y Pedraja Rejas, 2007) es posible plantear -al menos conceptualmenteque un mayor grado de conflicto de ideas debe conducir a decisiones más exhaustivamente elegidas (Rodríguez-Ponce, 2006). Gunter (2006), estudiando el campo de la educación superior, sugiere que la diversidad de ideas puede conducir a decisiones más racionales. Ideas previamente presentadas en trabajos de alto impacto, tales como Davies (2004) y Ensley y Hmieleski (2005).

La figura 1 representa, en forma integrada, el conjunto de hipótesis de la investigación: 
Figura 1: Modelo de la investigación

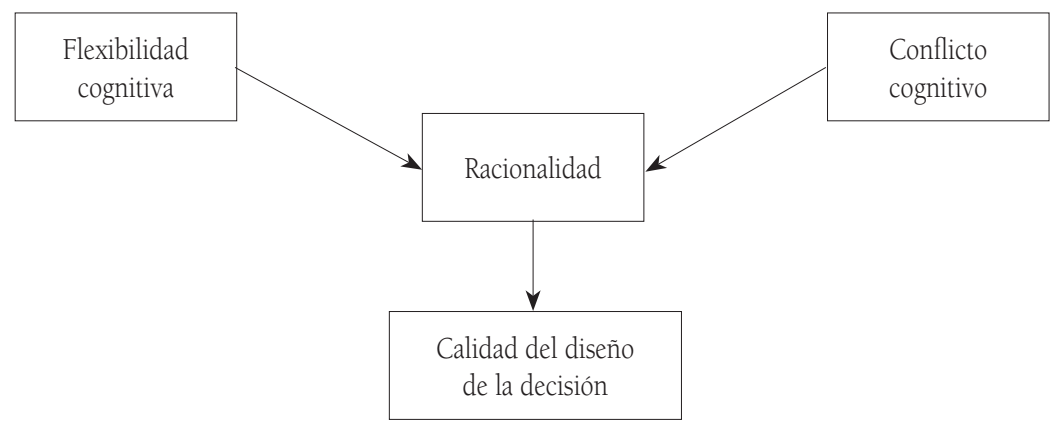

\section{Metodología}

\section{Muestra}

Se aplicó un cuestionario a 62 universidades chilenas y 62 universidades españolas. El cuestionario fue dirigido al rector y se solicitó expresamente que fuese respondido por éste o por un alto directivo que hubiese participado del proceso de toma de decisiones analizado. Al quinto mes se tenía respuesta de 25 universidades de España y 32 universidades de Chile. En esa fecha se envío una nueva carta a las instituciones que no habían dado respuesta, insistiendo en el requerimiento de respuesta a través de una nueva llamada telefónica.

Al cabo del séptimo mes se disponía de 43 respuestas de universidades chilenas y 35 de universidades españolas, de las cuales finalmente fueron utilizables un total de 71 (tasa de respuesta del $57,3 \%$ ), correspondientes a 32 universidades de España (tasa de respuesta del 51,6\%) y 39 de Chile (tasa del 69,2\%). Dichas tasas son elevadas si se comparan con estudios equivalentes (Pedraja Rejas y Rodríguez Ponce, 2008a; Rodríguez Ponce, 2007a, 2007b).

Las respuestas correspondieron, en forma agregada, a los siguientes altos directivos: 
Tabla 1: Altos directivos que respondieron el cuestionario

\begin{tabular}{|l|c|c|}
\hline Altos directivos & $\begin{array}{c}\text { Universidades } \\
\text { chilenas }\end{array}$ & $\begin{array}{c}\text { Universidades } \\
\text { españolas }\end{array}$ \\
\hline Rector & 20 & 9 \\
\hline $\begin{array}{l}\text { Vicerrector (Académico, Administración y Finanzas, otros } \\
\text { vicerrectores) }\end{array}$ & 11 & 11 \\
\hline $\begin{array}{l}\text { Directores superiores (Planificación y Desarrollo, Gestión de } \\
\text { Calidad, otros directores equivalentes) }\end{array}$ & 8 & 12 \\
\hline
\end{tabular}

Las decisiones analizadas fueron, en forma agregada, las siguientes:

Tabla 2: Decisiones estratégicas

\begin{tabular}{|l|c|c|}
\hline Decisiones & $\begin{array}{c}\text { Universidades } \\
\text { chilenas }\end{array}$ & $\begin{array}{c}\text { Universidades } \\
\text { españolas }\end{array}$ \\
\hline $\begin{array}{l}\text { Creación y/o fortalecimiento de programa de doctorado (incluye } \\
\text { alianzas estratégicas interuniversitarias). }\end{array}$ & 4 & 4 \\
\hline $\begin{array}{l}\text { Creación y/o fortalecimiento de programa de pregrado (incluye } \\
\text { modalidades de educación a distancia). }\end{array}$ & 6 & 2 \\
\hline $\begin{array}{l}\text { Creación y/o fortalecimiento de programas de extensión o } \\
\text { vinculación con el medio externo. }\end{array}$ & 3 & 5 \\
\hline Optimización de la estructura organizativa. & 5 & 4 \\
\hline $\begin{array}{l}\text { Optimización de procesos internos de tipo académico o } \\
\text { administrativo. }\end{array}$ & 5 & 4 \\
\hline Proceso de internacionalización. & 5 & 7 \\
\hline Apertura de nuevas sedes académicas. & 9 & 5 \\
\hline Otras decisiones estratégicas. & & \\
\hline
\end{tabular}

\section{Unidad y nivel de análisis}

La unidad de análisis fue una decisión recientemente implementada que haya involucrado una cantidad importante de recursos económicos para las instituciones; que proyectara efectos de largo plazo, que involucrara un alto número de funciones organizacionales y que tuviera alto impacto interno y externo. El nivel de análisis con el que se trabajó en este estudio fue el equipo de alta dirección.

\section{Variables y medidas}

La realización de estudios cuantitativos en el campo de las ciencias sociales y, más específicamente, en el ámbito de la toma de decisiones estratégicas en instituciones universitarias, plantea el problema de la medición de variables, su validez y fiabilidad. En este contexto, los 
protocolos internacionalmente adoptados y que prevalecen en las publicaciones de corriente principal suponen dos consideraciones fundamentales:

- La validez se puede abordar mediante el análisis de estudios previos de quienes hayan analizado variables equivalentes, en la perspectiva de incorporar y construir ítems previamente validados. Esta consideración está plenamente contemplada en la investigación, ya que cada una de las variables se diseña considerando mediciones e investigaciones previas; así por ejemplo:

- Calidad de la decisión, basada en Amason (1996). La variable se midió con una escala de Likert de 1 a 7 . Los ítems empleados fueron:

- La decisión adoptada:

- Fue de máxima calidad.

- Permitió el logro de los objetivos.

- Generó una solución eficaz y eficiente.

- Permitió crear valor y aportar al desarrollo de la universidad.

En forma adicional al trabajo de Amason (1996), la construcción de la variable calidad de la decisión basada en ítems equivalentes a los empleados en esta investigación ha sido validada consistentemente en múltiples investigaciones (Hollenbeck et al., 1998; Dean y Sharfman, 1996; Valacich y Schwenk, 1995; Molloy y Schwenk, 1995; Amason, 1996; Rodríguez Ponce, 2007a; 2007b; Pedraja Rejas et al., 2008a, 2008b).

- Racionalidad, basada en Dean y Sharfman (1993). La variable se midió con una escala de Likert de 1 a 7. Los ítems específicos empleados fueron:

- En el proceso de toma de decisiones estratégicas:

- La búsqueda y el análisis de información han sido profundos y exhaustivos.

- La generación y análisis de alternativas ha sido profundos y exhaustivos. 
- La selección de la opción ha sido rigurosa y basada en un criterio más analítico que intuitivo.

En forma adicional al trabajo de Dean y Sharfman (1993), la construcción de la variable racionalidad, basada en ítems equivalentes a los empleados en esta investigación, ha sido validada consistentemente en múltiples investigaciones (Dean y Sharfman, 1996; Papapadakis et al., 1998; Goll y Rasheed, 2005; Rodríguez Ponce, 2006; Rodríguez Ponce y Pedraja Rejas, 2007).

- Conflicto cognitivo, basada en Amason (1996). La variable se midió con una escala de Likert de 1 a 7. Los ítems empleados fueron:

- La decisión adoptada supuso:

- La discusión de muchas ideas diferentes.

- El análisis de múltiples perspectivas o enfoques diferentes.

- Muchas diferencias de opinión entre los participantes.

En forma complementaria al trabajo de Amason (1996), la construcción de la variable conflicto cognitivo, basada en ítems equivalentes a los empleados en esta investigación, ha sido validada consistentemente en múltiples investigaciones (Valacic y Schwenk, 1995; Jehn, 1997; Papadakis et al., 1998; Rodríguez Ponce y Pedraja Rejas, 2007).

- Flexibilidad cognitiva, basada en Sharfman y Dean (1997). La variable se midió con una escala de Likert de 1 a 7 . Los ítems específicos empleados fueron:

- En la decisión adoptada:

- Hubo posibilidad de incorporar nueva información en forma recurrente.

- Las ideas originales fueron bien acogidas.

- Los directivos que participaron lo hicieron en forma amplia y creativa más allá del ámbito de sus cargos.

- Se reconsideraron las posiciones iniciales. 
Conjuntamente con el trabajo de Sharfman y Dean (1997), la construcción de la variable flexibilidad cognitiva, basada en ítems equivalentes a los empleados en esta investigación, ha sido validada consistentemente en varias investigaciones (Dean y Sharfman, 1996; Rodríguez Ponce, 2004; Pinsirió, 2005; Rodríguez Ponce, 2006).

- La fiabilidad de las variables se mide de acuerdo con el cálculo del Alpha de Cronbach, el cual tiene como límite mínimo de aceptación el valor 0.70. Esta consideración está plenamente contemplada en esta investigación, ya que cada de las variables es estadísticamente fiable, como sugiere su Alpha de Cronbach:

- Calidad de la decisión, con un Alpha de Cronbach de $0.93>0.70$.

- Racionalidad, Alpha de Cronbach de $0.88>0.70$.

- Conflicto cognitivo, Alpha de Cronbach de $0.87>0.70$.

- Flexibilidad cognitiva, basada Alpha de Cronbach de $0.88>0.70$.

Lo que implica que la medición de estas variables es muy fiable en la presente investigación.

Tabla 3. Variables y medidas

\begin{tabular}{|l|l|c|c|}
\hline Variables & Ítems & Basado en & $\begin{array}{c}\text { Alpha de } \\
\text { Cronbach }\end{array}$ \\
\hline $\begin{array}{l}\text { Calidad de } \\
\text { la decisión }\end{array}$ & $\begin{array}{l}\text { La decisión adoptada: } \\
\text { - Fue de máxima calidad. } \\
\text { - Permitió el logro de los objetivos. } \\
\text { - Generó una solución eficaz y eficiente. } \\
\text { - Permitió crear valor y aportar al desarrollo de la } \\
\text { universidad. }\end{array}$ & $\begin{array}{l}\text { Amason } \\
(1996)\end{array}$ & 0.93 \\
\hline Racionalidad & $\begin{array}{l}\text { En el proceso de toma de decisiones estratégicas: } \\
\text { - La búsqueda y análisis de información han sido profundos } \\
\text { y exhaustivos. } \\
\text { - La generación y análisis de alternativas ha sido profunda } \\
\text { y exhaustiva. } \\
\text { - La selección de la opción ha sido rigurosa y basada en un } \\
\text { criterio más analítico que intuitivo. }\end{array}$ & $\begin{array}{c}\text { Sean y } \\
\text { (1993) }\end{array}$ & 0.88 \\
\hline $\begin{array}{l}\text { Conflicto } \\
\text { cognitivo }\end{array}$ & $\begin{array}{l}\text { La decisión adoptada supuso: } \\
\text { - La discusión de muchas ideas diferentes. } \\
\text { - El análisis de múltiples perspectivas o enfoques diferentes. } \\
\text { - Muchas diferencias de opinión entre los participantes. }\end{array}$ & $\begin{array}{l}\text { Amason } \\
(1996)\end{array}$ & 0.87 \\
\hline
\end{tabular}




\begin{tabular}{|c|c|c|c|}
\hline Flexibilidad & $\begin{array}{l}\text { En la decisión adoptada: } \\
\text { - Hubo posibilidad de incorporar nueva información en } \\
\text { forma recurrente. } \\
\text { - Las ideas originales fueron bien acogidas. } \\
\text { - Los directivos que participaron lo hicieron en forma } \\
\text { amplia y creativa más allá del ámbito de sus cargos. } \\
\text { - Se reconsideraron las posiciones iniciales. }\end{array}$ & $\begin{array}{c}\text { Sharfman } \\
\text { y Dean } \\
\text { (1997) }\end{array}$ & 0,88 \\
\hline
\end{tabular}

\section{Métodos}

Las relaciones establecidas en la figura 1 son verificadas mediante la aplicación de un modelo de regresión lineal simple para explicar la calidad de la decisión estratégica, según la siguiente ecuación de regresión:

$$
V x=\beta 0+a \beta 1+E i
$$

Donde Vx: variable dependiente, es decir, calidad de la decisión estratégica; $\beta 0$ : constante del modelo; a: variable independiente, es decir, racionalidad del proceso de toma de decisiones estratégicas; $\beta 1$ : factor de ponderación y Ei: error residual del modelo.

Para explicar la racionalidad del proceso de toma de decisiones estratégicas se aplica un modelo de regresión lineal múltiple, según la siguiente ecuación de regresión.

$$
V x=\beta 0+a \beta 1+b \beta 2+E i
$$

Donde Vx: variable dependiente, es decir, la racionalidad del proceso de toma de decisiones estratégicas; $\beta 0$ : constante del modelo; a: variable independiente denominada conflicto cognitivo; $\beta 1$ : factor de ponderación; b: variable independiente denominada flexibilidad cognitiva; $\beta 2$ : factor de ponderación y Ei: error residual del modelo.

\section{Resultados}

Los resultados obtenidos del análisis estadístico muestran las siguientes estadísticas descriptivas: 
Tabla 4: Estadísticas descriptivas

\begin{tabular}{|l|c|c|}
\hline Variables & Media & Desviación estándar \\
\hline Racionalidad & 4.79 & 1.03 \\
\hline Conflicto Cognitivo & 4.44 & 1.28 \\
\hline Flexibilidad & 4.78 & 1.14 \\
\hline Calidad de la decisión & 5.13 & 1.18 \\
\hline
\end{tabular}

En todos los casos los valores reales de las variables, tanto del proceso de toma de decisiones como de la calidad de la decisión, son significativamente diferentes del valor ideal siete $(p<0.01)$. Además, en todos los casos existe una variabilidad en las respuestas significativamente distinta de cero $(\mathrm{p}<0.01)$.

Los datos obtenidos muestran que los altos directivos no sólo han mostrado consistencia interna en sus respuestas (Alpha de Cronbach > 0.70), sino además la suficiente autocrítica para evaluarlas con ponderación y calificar en promedio racionalidad, conflicto cognitivo y flexibilidad en un nivel sólo regular, y promediando una calidad de las decisiones ligeramente sobre 5 , en escala de 1 a 7 .

Las tablas 5 y 6 muestran respectivamente las correlaciones de Pearson entre la racionalidad y la calidad de la decisión, así como los resultados de la aplicación de la ecuación de regresión lineal simple.

Tabla 5: Correlación de Pearson

\begin{tabular}{|l|c|c|}
\hline Variables & Diseño de la decisión & Racionalidad \\
\hline Calidad de la decisión & 1.000 & $0.854^{* * *}$ \\
\hline Racionalidad & 0.854 & 1.000 \\
\hline
\end{tabular}

Fuente: Procesamiento del cuestionario (*** implica que la correlación es significativa al 1\%).

Tabla 6: Resumen del modelo de regresión

\begin{tabular}{|l|c|c|c|c|c|}
\hline Concepto & $\begin{array}{c}\text { Coeficiente no } \\
\text { estandarizado }\end{array}$ & Error estándar & Test t & Significancia & R2 ajustado \\
\hline Constante & 1.219 & 0.295 & 4.136 & 0.000 & \\
\hline Racionalidad & 0.820 & 0.060 & 13.614 & 0.000 & \\
\hline Capacidad Explicativa & & & & & $0.725^{* * *}$ \\
\hline
\end{tabular}

Fuente: Procesamiento del cuestionario (*** implica que la capacidad explicativa del modelo es significativa al 1\%). 
Los resultados muestran que la racionalidad del proceso de toma de decisiones estratégicas es un determinante estructural de la calidad de la decisión diseñada. Su capacidad explicativa llega al $72,5 \%$ ( $\mathrm{p}<0.01$ ), y su significancia estadística es muy alta (Test $\mathrm{t}=$ 13.614; $\mathrm{p}<0.01$ ), aunque lógicamente este modelo simple omite variables (Test t de la constante $=4.136 ; \mathrm{p}<0.01$ ).

La tablas 7 y 8 muestran las correlaciones de las variables involucradas en la investigación y los resultados de la aplicación de la ecuación de regresión lineal múltiple, respectivamente.

Tabla 7: Correlación de Pearson

\begin{tabular}{|l|c|c|c|}
\hline Variables & Racionalidad & Conflicto cognitivo & Flexibilidad cognitiva \\
\hline Racionalidad & 1.000 & $0.705^{* * *}$ & $0.847^{* * *}$ \\
\hline Conflicto cognitivo & 0.705 & 1.000 & 0.557 \\
\hline Flexibilidad cognitiva & 0.847 & 0.557 & 1.000 \\
\hline
\end{tabular}

Fuente: Procesamiento del cuestionario (*** implica que la correlación es significativa al 1\%).

Tabla 8: Resumen del modelo de regresión

\begin{tabular}{|l|c|c|c|c|c|}
\hline Concepto & $\begin{array}{c}\text { Coeficiente no } \\
\text { estandarizado }\end{array}$ & $\begin{array}{c}\text { Error } \\
\text { estándar }\end{array}$ & Test t & Significancia & $\begin{array}{c}\text { R2 } \\
\text { ajustado }\end{array}$ \\
\hline Constante & 0.736 & 0.255 & 2.889 & 0.005 & \\
\hline Conflicto cognitivo & 0.271 & 0.053 & 5.121 & 0.000 & \\
\hline Flexibilidad cognitiva & 0.595 & 0.059 & 10.001 & 0.000 & \\
\hline Capacidad Explicativa & & & & & $0.790 * * *$ \\
\hline
\end{tabular}

Fuente: Procesamiento del cuestionario (*** implica que el modelo de regresión es significativo al $1 \%)$.

Los resultados muestran que el conflicto cognitivo (Test $\mathrm{t}=$ 5.121, p < 0.01) y la flexibilidad cognitiva (Test t 10.001, p < 0.01) son determinantes estructurales de la racionalidad del proceso de toma de decisiones estratégicas. Su capacidad explicativa llega al $79,0 \%(\mathrm{p}<0.01)$. De todas maneras, el modelo omite variables relevantes (Test t de la constante $=2.889, \mathrm{p}<0.01$ ).

El análisis integrado de los modelos de regresión simple y múltiple dan soporte a la figura 1 y a cada una de las hipótesis de la investigación, como se resume en la tabla 9: 
Tabla 9: Resumen del contraste de las hipótesis de la investigación

\begin{tabular}{|l|l|l|}
\hline Hipótesis & Resultados & Aceptación / Rechazo \\
\hline $\begin{array}{l}\text { La racionalidad impacta } \\
\text { positivamente sobre el diseño } \\
\text { de las decisiones estratégicas. }\end{array}$ & $\begin{array}{l}\text { Regresión simple: } \\
\text { Correlación de Pearson } 0.854(\mathrm{p}<0.01) .\end{array}$ & Aceptación \\
Test $\mathrm{t}=13.614(\mathrm{p}<0.01)$. & \\
\hline $\begin{array}{l}\text { El conflicto cognitivo } \\
\text { impacta positivamente sobre } \\
\text { el grado de racionalidad } \\
\text { del proceso de toma de } \\
\text { decisiones estratégicas. }\end{array}$ & $\begin{array}{l}\text { Regresión múltiple: } \\
\text { Correlación de Pearson } 0.705(\mathrm{p}<0.01) .\end{array}$ & Aceptación \\
\hline $\begin{array}{l}\text { R2 ajustado }=79.0 \%(\mathrm{p}<0.01) . \\
\text { Test } \mathrm{t}=5.121(\mathrm{p}<0.01) .\end{array}$ & \\
$\begin{array}{l}\text { impacta positidad cognitiva } \\
\text { el grado de racionalidad } \\
\text { del proceso de toma de } \\
\text { decisiones estratégicas. }\end{array}$ & $\begin{array}{l}\text { Regresión Múltiple: } \\
\text { Correlación de Pearson } 0.847(\mathrm{p}<0.01) .\end{array}$ & \\
\hline
\end{tabular}

Limitaciones y alcances de la investigación

La medición de las variables aplicando preguntas de cuestionario a un mismo sujeto en el mismo momento es una debilidad de la metodología empleada. Esta forma de obtención de información puede sobrevaluar las relaciones entre las variables en función de la búsqueda de coherencia en las respuestas por parte del encuestado. En consecuencia, asumiendo la lógica de Katouzian (1980), la investigación sólo pretende entregar antecedentes empíricos de las relaciones entre flexibilidad cognitiva, conflicto cognitivo, racionalidad y calidad del diseño de las decisiones estratégicas para el conjunto de instituciones analizadas. Siguiendo la lógica de Snow y Thomas (1994), se debe indicar que en este estudio empírico no se pretende construir teoría ni medir la capacidad predictiva de un modelo, sino que, más bien, proporcionar evidencia empírica incremental que contribuya al conocimiento del estado del arte, al identificar relaciones estadísticamente significativas en la muestra de estudio.

Trabajos de amplia aceptación internacional cuentan con limitaciones equivalentes. Papadakis et al. (1998) tiene 128 citaciones; Dean y Sharfman (1993) tiene 101; Priem et al. (1995) tiene 101 citaciones, entre otros similares. 


\section{Conclusiones}

Una primera conclusión es que la racionalidad del proceso de toma de decisiones estratégicas impacta positivamente sobre su calidad. Este hallazgo es consistente con la literatura dominante sobre el tema (Goll y Rasheed, 2005; Miller et al., 1998; Papadakis et al., 1998).

Una segunda conclusión consiste en un hallazgo sugerido conceptualmente (Sharfman y Dean, 1997) pero escasamente demostrado en el estado del arte: la flexibilidad cognitiva impacta positiva y significativamente sobre el grado de racionalidad del proceso de toma de decisiones estratégicas.

En tercer término, es empíricamente posible demostrar que el conflicto de ideas contribuye a lograr mayores niveles de racionalidad en las decisiones estratégicas, conclusión también sugerida por Rodríguez-Ponce (2006).

En consecuencia, a modo de buenas prácticas y para lograr mayores niveles de éxito en la toma de decisiones estratégicas, los equipos de alta dirección deben:

- Favorecer la racionalidad de las decisiones estratégicas, es decir:

- Buscar y analizar información en forma profunda y exhaustiva.

- Generar alternativas en forma exhaustiva.

- Realizar una selección rigurosa y analítica de la opción estratégica.

Lo cual se logrará en mayor medida en tanto los equipos de alta dirección:

- Favorezcan la flexibilidad cognitiva en el proceso de toma de decisiones estratégicas, es decir, estimulen:

- La discusión de muchas ideas diferentes.

- El análisis de múltiples perspectivas o enfoques diferentes.

- Las diferencias de opinión entre los participantes.

- La incorporación de nueva información en forma recurrente. 
- Propicien el conflicto cognitivo en el proceso de toma de decisiones estratégicas, es decir, estimulen:

- La discusión de nuevas ideas

- La recepción de ideas originales.

- Participar en forma amplia y creativa más allá del ámbito de sus cargos.

- Reconsiderar las posiciones o consensos iniciales.

\section{Referencias bibliográficas}

Amason, A. (1996) Distinguishing the effects of functional and dysfunctional conflict on strategic decision-making: Resolving a paradox for top management teams. Academy of Management Journal, 39, pp. 123148.

Arendt, L., Priem, R., Ndofor, H. (2005) A CEO-Adviser Model of Strategic Decision Making. Journal of Management, 31, pp. 680-699.

Athaide, G., Desai, H. (2005) Design and Implementation of an Interdisciplinary Marketing/Management Course on Technology and Innovation Management. Journal of Marketing Education, 27, pp. 239-249.

Carpenter, M., Fredrickson, J. (2001) Top management teams, global, strategy posture, and the moderating role of uncertainty. Academy of Management Journal, 4, pp. 533-545.

Changchien, S., Lin, M. (2005) Design and implementation of a case-based reasoning system for marketing plans. Expert systems with applications, 28, pp. 43-53.

Davies, L. (2004) Education and Conflict: Complexity and Chaos. Routledge.

De Boer, H., Enders, J., Leisyte, L. (2007) Public sector reform in dutch higher education: the organizational transformation of the university. Public Administration, 85, pp. 27-46.

Dean, J., Sharfman, M. (1993) Procedural Rationality in strategic decisionmaking process. Journal of Management Studies, 30, pp. 587-610.

Dean, J., Sharfman, M. (1996) Does decision process matter? A study of strategic decision making effectiveness. Academy of Management Journal, 39, pp. 368-396.

Dowlatshahi, S. (2005) A strategic framework for the design and implementation of remanufacturing operations in reverse logistics. International Journal of Production Research, 43, pp. 3455-3480. 
Ensley, M., Hmieleski, K. (2005) A comparative study of new venture top management team composition, dynamics and performance between university-based and independent start-ups. Research Policy, 34, pp. 1091-1105.

Goll, I., Rasheed, A. (1997) Rational decision-making and firm performance: The moderating role of environment. Strategic Management Journal, 18, pp. 583-591.

Goll, I., Rasheed, A. (2005) The relationships between top management demographic characteristics, rational decision making, environmental munificence, and firm performance. Organization Studies, 26, pp. 999-1023.

Grover, V., Segars, A. (2005) An empirical evaluation of stages of strategic information systems planning: patterns of process design and effectiveness. Information \& Management, 42, pp. 761-779.

Gunter, H. (2006) Educational Leadership and the Challenge of Diversity. Educational Management Administration \& Leadership, 34, pp. 257-268.

Hambrick, D. y Mason, P. (1984) Upper echelons: The organization as a reflection of its top managers. Academy of Management Review, 9, pp. 193-206.

Hambrick, D. y Mason, P. (2007) Upper echelons theory: an update. The Academy of Management Review, 32, pp. 334-343.

Hatcher, R. (1998) Class Differentiation in Education: rational choices? British Journal of Sociology of Education, 19, pp. 5-24.

Hiller, N., Hambrick, D. (2005) Conceptualizing executive hubris: the role of (hyper-) core self-evaluations in strategic decision-making. Strategic Management Journal, 26, pp. 297-319.

Jehn, K. (1997) A multimethod examination of the benefits and detriments of intragroup conflict. Administrative Science Quarterly, 42, pp. 530-557.

Katouzian, H. (1980) Ideology and method in economics. London: MacMillan; 1980.

Katsikeas, C., Samiee, S., Theodosiou, M. (2006) Strategy fit and performance consequences of international marketing standardization. Strategic Management Journal, 27, pp. 867-890.

Leader, G. (2004) Further Education Middle Managers. Educational Management Administration \& Leadership, 32, pp. 67-79.

Miller, C., Burke, L., Glick, W. (1998) Cognitive diversity among upper echelon executives: Implications for strategic decision processes. Strategic Management Journal, 19, pp. 39-58. 
Molloy, S., Schwenk, C. (1995) The effects of information technology on strategic decision making. Journal of Management Studies, 32, pp. 283-311.

Nutt, P.C. (1999) Surprising but true: Half the decisions in organizations fail. The Academy of Management Executive, 13, pp. 75-90.

Pansiri, J. (2005) The influence of managers' characteristics and perceptions in strategic alliance practice. Management Decision, 43, pp. 1097-1113.

Papadakis, V., Lioukas, S., Chambers, D. (1998) Strategic decision-making processes: the role of management and context. Strategic Management Journal, 19, pp. 115-147.

Pedraja Rejas, L., Rodríguez Ponce, E., Rodríguez Ponce, J. (2008) The influence of leadership styles on effectiveness: A comparative study among large, small and medium-sized private business. Rev. Cienc. Soc., 14, pp. 21-29.

Pedraja Rejas, L., Rodríguez Ponce, E. (2008a) Estudio comparativo de la influencia del estilo de liderazgo y la congruencia de valores en la eficacia de empresas privadas e instituciones públicas. Interciencia, 33, pp. 8-13.

Pedraja Rejas, L., Rodríguez Ponce, E. (2008b) Estilos de liderazgo, gestión del conocimiento y diseño de la estrategia: un estudio empírico en pequeñas y medianas empresas. Interciencia, 33, pp. 651-657.

Priem, R., Rasheed, A., Kotulic, A. (1995) Rationality in Strategic Decision Processes. Environmental Dynamism and Firm Performance. Journal of Management, 21, pp. 913-929.

Rodríguez Ponce, E. (2004) La toma de decisiones estratégicas: una perspectiva integradora. Santiago de Chile: Ediciones Consorcio de Universidades Estatales.

Rodríguez Ponce, E. (2006) El proceso de la toma de decisiones estratégicas en las universidades públicas. Revista Calidad en la Educación, 24, pp. 49-63.

Rodríguez Ponce, E. (2007a) Gestión del conocimiento y eficacia de las organizaciones: Un estudio empírico en instituciones públicas. Interciencia, 32, pp. 820-826.

Rodríguez Ponce, E. (2007b) Leadership styles, strategic decision making, and performance: An empirical study in small and medium firms. Interciencia, 32, pp. 522-528.

Rodríguez Ponce, E., Pedraja Rejas, L. (2007) Efectos e implicaciones de las decisiones estratégicas en las instituciones universitarias. Interciencia, 32, pp. 593-600. 
Rodríguez Ponce, E., Rodríguez Ponce, J. (2007) La toma de decisiones estratégicas en las pequeñas y mediana empresas. Soc. Inf., 8, pp. 5-8.

Sharfman, M., Dean J. (1997) Flexibility in strategic decision making. Journal of Management Studies, 34, pp. 191-217.

Snow, C., Thomas, J. (1994) Fields research methods in strategic management contributions to the building and testing. Journal of Management Studies, 31 , pp. $457-480$

Tapinos, E., Dyson, R., Meadows, M. (2005) The impact of performance measurement in strategic planning. International Journal of Productivity and Performance Management, 54, pp. 370-384.

Valacich, J.S., Schwenk, C. (1995) Structuring conflict in individual, face-toface, and computer-mediated group decision making: Carping versus objective devil's advocacy. Decision Science, 26, pp. 369-393.

Waldman, D., Ramírez, G., House, R., Puranam, P. (2001) Does leadership matter? CEO leadership attributes and profitability under conditions of perceived environmental uncertainty. Academy of Management Journal, 44, pp. 134-143.

Recibido: 15 de julio de 2008

Aceptado: 22 de octubre de 2008 


\section{Anexo \\ Cuestionario}

Identificación de la universidad

Nombre:

Dirección:

Datos del encuestado

Persona que responde el cuestionario:

Cargo:

Instrucciones para responder:

Para responder el cuestionario tenga presente las siguientes consideraciones:

1. Seleccione una decisión estratégica recientemente implementada que se ha caracterizado debido a:

2. Todas las preguntas hacen referencia a la decisión estratégica seleccionada por usted.

3. En cada pregunta encierre en un círculo la respuesta que mejor representa su opinión o evaluación.

4. La escala de medición de sus respuestas es creciente, en la cual 1 significa total desacuerdo y 7 significa total acuerdo.

Decisión estratégica:

Preguntas:

1. La decisión analizada se ha basado en un proceso de búsqueda profunda y exhaustiva de la información

\begin{tabular}{|l|l|l|l|l|l|l|}
\hline 1 & 2 & 3 & 4 & 5 & 6 & 7 \\
\hline
\end{tabular}

2. La decisión analizada ha requerido un análisis en profundidad de la información relevante, generándose alternativas en forma exhaustiva.

\begin{tabular}{|l|l|l|l|l|l|l|}
\hline 1 & 2 & 3 & 4 & 5 & 6 & 7 \\
\hline
\end{tabular}

3. La evaluación de las alternativas ha sido rigurosa y exhaustiva.

\begin{tabular}{|l|l|l|l|l|l|l|}
\hline 1 & 2 & 3 & 4 & 5 & 6 & 7 \\
\hline
\end{tabular}

4. La selección de la decisión ha sido rigurosa y se ha basado más en el criterio analítico que en la intuición de los actores.

\begin{tabular}{|l|l|l|l|l|l|l|}
\hline 1 & 2 & 3 & 4 & 5 & 6 & 7 \\
\hline
\end{tabular}


5. La decisión analizada ha supuesto la discusión de múltiples ideas diferentes.

\begin{tabular}{|l|l|l|l|l|l|l|}
\hline 1 & 2 & 3 & 4 & 5 & 6 & 7 \\
\hline
\end{tabular}

6. La decisión analizada ha supuesto el análisis de múltiples perspectivas o enfoques.

\begin{tabular}{|l|l|l|l|l|l|l|}
\hline 1 & 2 & 3 & 4 & 5 & 6 & 7 \\
\hline
\end{tabular}

7. La decisión analizada ha supuesto la existencia de diferencias de opinión entre los participantes.

\begin{tabular}{|l|l|l|l|l|l|l|}
\hline 1 & 2 & 3 & 4 & 5 & 6 & 7 \\
\hline
\end{tabular}

8. En el proceso de adopción de la decisión se confió en nuevas fuentes de información en forma frecuente.

\begin{tabular}{|l|l|l|l|l|l|l|}
\hline 1 & 2 & 3 & 4 & 5 & 6 & 7 \\
\hline
\end{tabular}

9. En el proceso de adopción de la decisión analizada las ideas originales fueron bien recibidas.

\begin{tabular}{|l|l|l|l|l|l|l|}
\hline 1 & 2 & 3 & 4 & 5 & 6 & 7 \\
\hline
\end{tabular}

10. Los directivos que participaron en la decisión tuvieron una contribución amplia y creativa, más allá de las materias definidas por su cargo.

\begin{tabular}{|l|l|l|l|l|l|l|}
\hline 1 & 2 & 3 & 4 & 5 & 6 & 7 \\
\hline
\end{tabular}

11. En el proceso de la adopción de la decisión se dio lugar a reconsiderar algunas posiciones iniciales.

\begin{tabular}{|l|l|l|l|l|l|l|}
\hline 1 & 2 & 3 & 4 & 5 & 6 & 7 \\
\hline
\end{tabular}

12. La calidad de la decisión adoptada es la máxima que se podía lograr.

\begin{tabular}{|l|l|l|l|l|l|l|}
\hline 1 & 2 & 3 & 4 & 5 & 6 & 7 \\
\hline
\end{tabular}

13. La decisión adoptada permite el logro de los objetivos que se esperaba cumplir con dicha decisión.

\begin{tabular}{|l|l|l|l|l|l|l|}
\hline 1 & 2 & 3 & 4 & 5 & 6 & 7 \\
\hline
\end{tabular}

14. La decisión adoptada presenta una solución eficaz y eficiente a la problemática a resolver con el proceso decisional.

\begin{tabular}{|l|l|l|l|l|l|l|}
\hline 1 & 2 & 3 & 4 & 5 & 6 & 7 \\
\hline
\end{tabular}

15. La decisión adoptada ha creado valor académico, económico o estratégico y permitió aportar al desarrollo de la universidad.

\begin{tabular}{|l|l|l|l|l|l|l|}
\hline 1 & 2 & 3 & 4 & 5 & 6 & 7 \\
\hline
\end{tabular}

